\title{
An improved Xer-cise technology for the generation of multiple unmarked mutants in Mycobacteria
}

Yves-Marie Boudehen', Maximilian Wallat', Philippe Rousseau², Olivier Neyrolles' \& Claude Gutierrez*,1

\section{ABSTRACT}

Xer-cise is a technique using antibiotic resistance cassettes flanked by dif sites allowing spontaneous and accurate excision from bacterial chromosomes with a high frequency through the action of the cellular recombinase XerCD. Here, we report a significant improvement of Xer-cise in Mycobacteria. Zeocin resistance cassettes flanked by variants of the natural Mycobacterium tuberculosis dif site were constructed and shown to be effective tools to construct multiple unmarked mutations in $M$. tuberculosis and in the model species Mycobacterium smegmatis. The dif site variants harbor mutations in the central region and can therefore not recombine with the wild-type or other variants, resulting in mutants of increased genetic stability. The herein described method should be generalizable to virtually any transformable bacterial species.

\section{METHOD SUMMARY}

dif-Zeo ${ }^{R}$-dif cassettes are used to replace non-essential genes in mycobacterial genome through recombineering. Spontaneous excision of the cassette is carried out under the action of the recombinase XerCD, resulting in unmarked deletions. Subsequent rounds of mutagenesis using cassettes flanked by a range of dif site variants allow construction of multiple mutants in which the different dif sites cannot recombine with each other, yielding stable genetic constructs.

\section{KEYWORDS}

dif site $\cdot$ excisable cassette $\cdot$ mutagenesis - Mycobacterium • recombineering • unmarked deletions $\cdot$ Xer-cise

'Institut de Pharmacologie et de Biologie Structurale (IPBS), Université de Toulouse, CNRS, UPS, 205 route de Narbonne, F-31400 Toulouse, France; ${ }^{2}$ Centre de Biologie Intégrative de Toulouse (CBI-Toulouse), Laboratoire de Microbiologie et de Génétique Moléculaires (LMGM), Université de Toulouse, CNRS, UPS, F-31400 Toulouse, France; *Author for correspondence: claude.gutierrez@ipbs.fr

BioTechniques 68: 106-110 (February 2020) 10.2144/ btn-2019-0119
Genetic studies of bacterial evolution and physiology require efficient and reliable tools for the construction of specific gene deletion mutants. A range of tools are available to manipulate Mycobacteria, such as Mycobacterium tuberculosis, the etiologic agent of tuberculosis. In particular, the development of recombineering has been a considerable improvement over previous methods [1]. This technique allows replacement of a specific gene by an antibiotic resistance cassette through homologous recombination between linear DNA fragments, so-called allelic exchange substrates (AES) and chromosomal DNA catalyzed by RecET-like mycobacteriophage proteins gp60 and gp61 (Figure 1D). However, only a limited number of selectable marker genes are available for mycobacteria [2] and, for safety reasons, strains used for medical studies must not carry resistance to multiple antibiotics.

One way to overcome these problems is to use excisable cassettes that can be removed by recombination between two specific sites repeated at both sides of the cassette. The so-called Xer-cise technique is a convenient way to achieve auto-excision, because the recombination between two deletion-induced filamentation (dif) sites is catalyzed by the XerCD recombinase present and active in most bacterial species, as it is required for chromosome maintenance [3]. Xer-cise has been adapted to Mycobacteria and shown to be efficient both in M. tuberculosis and in the fastgrowing model species Mycobacterium smegmatis $[2,4,5]$. However, these studies used the wild-type dif sequence of Mycobacteria [4,6], leading to two possible drawbacks. First, XerCDmediated recombination may occur between the dif sites on the AES and the endogenous chromosomal dif site, which may generate false positives during the selection of antibiotic resistant recombinant clones. Second, after completion of the recombination events, the presence of several copies of the dif site on the bacterial chromosome may be deleterious because recombination between these sites may generate deletions or inversions on the chromosome, depending on the relative orientation of the dif sites [7].

Here, we report an improvement of the Xer-cise technique in Mycobacteria. We designed and validated a set of variants of the natural $M$. tuberculosis dif site (Table 1) that can recombine with another copy of the same sequence, prompting excision of the antibiotic resistance cassette, but should recombine neither with the wild-type nor with the other variant dif sites, yielding stable, unmarked multiple mutants.

\section{DIF SITE VARIANTS}

dif sites have been identified in M. tuberculosis and $M$. smegmatis $[4,6]$. The minimal site is 28-bp long, with a XerCbinding left arm, a XerD-binding right arm and a 6-bp central region (Table 1). The mechanism of XerCD-mediated recombination has been well described in Escherichia coli $[8,9]$. It involves the formation of a synaptic complex gathering two dif sites in a head-to-tail conformation inside a XerCD hetero-tetramer. The two XerD protomers create single-strand cleavage on each dif site, at the border of the central region, then promote strand transfer at the equivalent positions of the other site, leading to a Holliday junctioncontaining intermediate. By analogy with other site-specific recombinases of the Y-recombinase family [8], efficient strand transfer requires that the first two to three nucleotides of the central region can pair. Indeed, recombination between sites with heterologous central region is inefficient $[10,11]$. After isomerization of the 


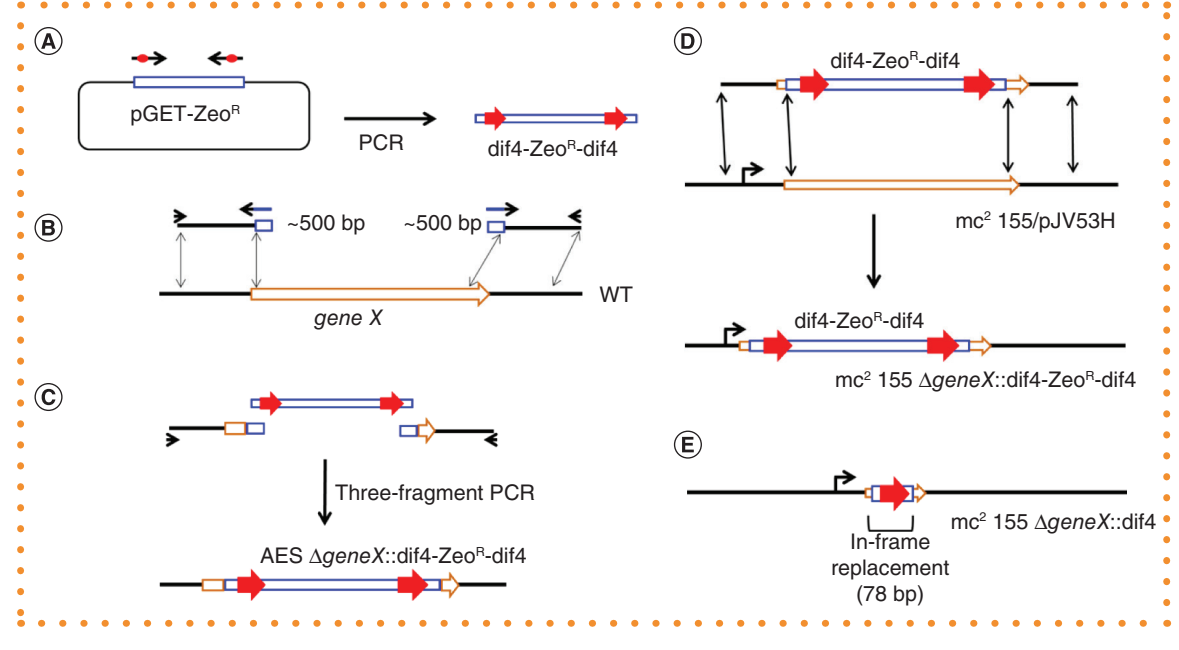

intermediate, XerC protomers cleave both dif partners on the other side of the central region and transfer the second pair of strands by the same mechanism, allowing resolution of the intermediate and completion of the reaction. Both steps of reciprocal strand transfer require complementary sequences in the two recombination partners. Therefore, concerted modifications of the first two nucleotides on both sides of the central region result in dif site variants that are still able to recombine with an identical site, because complementarity is conserved, but not with the wild-type sequence with which base pairing at the border of the central region is no longer possible. Four variants, named dif4 to dif7, were designed following this rationale (Table 1). These variants should not be able to recombine with each other, allowing construction of stable strains carrying multiple variants in their genome. Using specific oligonucleotides (Table 2) and plasmid pGET-Zeo $^{\mathrm{R}}$ as a template, we amplified by PCR a set of Zeocin-resistance cassettes [12] flanked by the four variants of the dif site (Figure 1A).

\section{STRATEGY TO CONSTRUCT UNMARKED DELETED MUTANTS}

To delete a gene of interest, 500-bp-long regions of homology corresponding to the flanking upstream and downstream sequences were amplified by PCR using oligonucleotides that introduce sequences overlapping the right or left end of the dif-Zeo $^{\mathrm{R}}$-dif cassettes, outside the dif sites (Figure 1B, Table 2, Supplementary Tables 1 $\& 2)$. Next, these fragments were mixed with one of the dif-Zeo ${ }^{\mathrm{R}}$-dif cassettes for a threefragment PCR, allowing amplification of an 1700-bp AES (Figure 1B \& C). The purified AES were used to transform $M$. tuberculosis (strain H37Rv, ATCC27294) or M. smegmatis (strain mc ${ }^{2} 155$, ATCC700084) carrying the plasmid pJV53H, a derivative of pJV53 [1], which expresses a mycobacteriophage

\section{Table 1. Sequence of wild-type and variant dif sites ${ }^{\dagger}$.}

\begin{tabular}{|l|l|}
\hline dif site & Sequence \\
\hline M. smegmatis dif $^{+}$ & AGTACCGATAA GCTACA TTATGTCAACT \\
\hline M. tuberculosis dif ${ }^{+}$ & TAAGCCGATAA GCGACA TTATGTCAAGT \\
\hline M. tuberculosis dif4 & TAAGCCGATAA ATGATG TTATGTCAAGT \\
\hline M. tuberculosis dif5 & TAAGCCGATAA TAGAGT TTATGTCAAGT \\
\hline M. tuberculosis dif6 & TAAGCCGATAA CGGAAC TTATGTCAAGT \\
\hline M. tuberculosis dif7 & TAAGCCGATAA CTGATC TTATGTCAAGT \\
\hline $\begin{array}{l}{ }^{+} \text {Left and right arm of the 28-bp dif sites are shown in black. The 6-bp central sequence is } \\
\text { shown in red. Nucleotides modified in each variant are in bold letters. }\end{array}$ \\
\hline
\end{tabular}

Figure 1. Construction of unmarked deletions in Mycobacteria by recombineering using zeocinresistance cassettes flanked by dif site variants. (A) PCR amplification of $Z^{2} o^{R}$ cassettes with oligonucleotides introducing dif site variants on each side (red arrows). (B) PCR amplification of DNA fragments flanking the gene to be deleted. Blue boxes show sequence extensions identical to the left or right ends of the Zeo ${ }^{R}$ cassette. (C) Three-fragment PCR amplification yielding the AES. (D) Recombineering in Mycobacteria carrying plasmid pJV53H yields $Z^{2} o^{R}$ recombinant clones. (E) Spontaneous XerCD-dependent recombination between the tandem dif site variants generates unmarked deletion with a 78-bp scar replacing the deleted sequences. AES: Allelic exchange substrate.

recombinase and confers resistance to hygromycin (Figure 1D\&E). Transformation protocol was performed as described [1], performed with $100 \mathrm{ng}$ of AES DNA, as recommended. Transformants were plated on Middlebrook 7H11 agar (Difco, Detroit, MI) supplemented with $10 \%$ oleic acidalbumin-dextrose-catalase (Difco) (complete $7 \mathrm{H} 11$ medium) and zeocin $(25 \mu \mathrm{g}$ $\mathrm{ml}^{-1}$; Thermo Fisher Scientific, MA, USA). Zeocin-resistant $\left(Z e o^{R}\right)$ clones were restreaked on the same medium, and single $Z_{e o^{R}}$ colonies were inoculated in Middlebrook $7 \mathrm{H} 9$ medium (Difco) supplemented with $10 \%$ albumin-dextrose-catalase (Difco) and 0.05\% Tween 80 (complete $7 \mathrm{H} 9$ medium) without antibiotic for growth, DNA extraction and verification by PCR. Zeo ${ }^{R}$ clones exhibited replacement of the band corresponding to the wild-type sequence by bands of the expected size for the dif-Zeo ${ }^{\mathrm{R}}$-dif cassette insertion. Several of these clones were streaked on complete $7 \mathrm{H} 11$ agar without antibiotic and grown at $37^{\circ} \mathrm{C}$. Fifty single colonies were then phenotypically tested in grids on complete $7 \mathrm{H} 11$ agar, with or without zeocin, to screen for spontaneous excision of the cassette. At this stage, the number of $\mathrm{Zeo}^{\mathrm{s}}$ colonies appeared variable from clone to clone with the same dif variant, presumably depending on how early excision events had occurred during the preculture of the candidates. However, we systematically observed $Z \mathrm{oo}^{\mathrm{s}}$ among the 50 clones tested, regardless of the dif variant used, demonstrating that all of the variants are functional and can recombine with efficiencies falling into the same range. $\mathrm{Zeo}^{\mathrm{S}}$ clones were inoculated 
Table 2. Oligonucleotides used to amplify Zeocin resistance cassette flanked by variant dif sites ${ }^{\dagger}$.

\begin{tabular}{|c|c|}
\hline Primers & Sequence $\left(5^{\prime}\right.$ to $\left.3^{\prime}\right)$ \\
\hline Zeo-Dif4-Fw & СTCCACGTGGATCGACTGCCAGGCACTTGACATAACATCATTTATCGGCTTAATCAAATAAAACGAAAGGCTC \\
\hline Zeo-Dif4-Rv & GAGCACGTGGGTCTGACGCTCAGTGGTAAGCCGATAAATGATGTTATGTCAAGTAACGAAAACTCACGTTAAGGG \\
\hline Zeo-Dif5-Fw & СTCCACGTGGATCGACTGCCAGGCACTTGACATAA ACTCTATTATCGGCTTAATCAAATAAAACGAAAGGCTC \\
\hline Zeo-Dif5-Rv & GAGCACGTGGGTCTGACGCTCAGTGGTAAGCCGATAATAGAGTTTATGTCAAGTAACGAAAACTCACGTTAAGGG \\
\hline Zeo-Dif6-Rv & GAGCACGTGGGTCTGACGCTCAGTGGTAAGCCGATAACGGAACTTATGTCAAGTAACGAAAACTCACGTTAAGGG \\
\hline Zeo-Dif7-Fw & CTCCACGTGGATCGACTGCCAGGCACTTGACATAA GATCAGTTATCGGCTTAATCAAATAAAACGAAAGGCTC \\
\hline Zeo-Dif7-Rv & GAGCACGTGGGTCTGACGCTCAGTGGTAAGCCGATAACTGATCTTATGTCAAGTAACGAAAACTCACGTTAAGGG \\
\hline
\end{tabular}

'Sequence corresponding to the dif site are shown in bold letters. Red letters show the nucleotides modified in each variant of dif.

in complete $7 \mathrm{H} 9$ medium for growth, DNA extraction and PCR verification. For construction of multiple mutants, the verified $\mathrm{Zeo}^{\mathrm{S}} \mathrm{Hyg}^{\mathrm{R}}$ clones were submitted to a subsequent round of mutagenesis, using a dif-Zeo ${ }^{\mathrm{R}}$ - dif cassette with a different dif site variant. Finally, clones verified to carry the expected mutation(s) were streaked on complete $7 \mathrm{H} 11$ agar without antibiotic, and single colonies were pheno-

Figure 2. Verification of the quadruple mutant of $M$. smegmatis $\mathrm{mc}^{2} 155 \Delta$ MSMEG_3155::dif6 $\triangle M S M E G \_1517::$ dif4 $\triangle$ MSMEG_4576::dif5 $\triangle$ MSMEG_6059-6058::dif7. (A) Schematics of the primers (arrowheads) and PCR products used to verify the four deletions. Brown arrowheads indicate primers internal to the sequences to be deleted. Red numbers in front of each pair of primers refer to the lanes in (B). (B) Agarose gel analysis of the amplified fragments. PCR was performed using DNA from wild-type $\mathrm{mc}^{2} 155$ (odd numbers) or the triple mutant strain (even numbers).

M: 1 kb DNA ladder (Thermo Scientific, MD, USA), bands of higher intensity correspond to 1 , 3 and $6 \mathrm{kbp}$. typically tested in grids on complete $7 \mathrm{H} 11$ agar, with or without hygromycin, to screen for spontaneous loss of the pJV53H plasmid $\left(\mathrm{Hyg}^{\mathrm{S}}\right)$. The efficiency of this step could be improved by the use of a counterselection marker or a visual phenotype screening associated to plasmid pJV53H, as described [5]. A final DNA extraction and PCR verification of each mutant was performed on the $\mathrm{Zeo}^{\mathrm{s}} \mathrm{Hyg}^{\mathrm{s}}$ clones
(Figures $2 \& 3$ )

\section{CONSTRUCTION OF MULTIPLE UNMARKED DELETIONS IN M. SMEGMATIS}

To validate the functionality of this system, we constructed deletion mutants in M. smegmatis $\mathrm{mc}^{2} 155$. Successive rounds of recombineering, using AES constructed with appropriate primers (Supple-

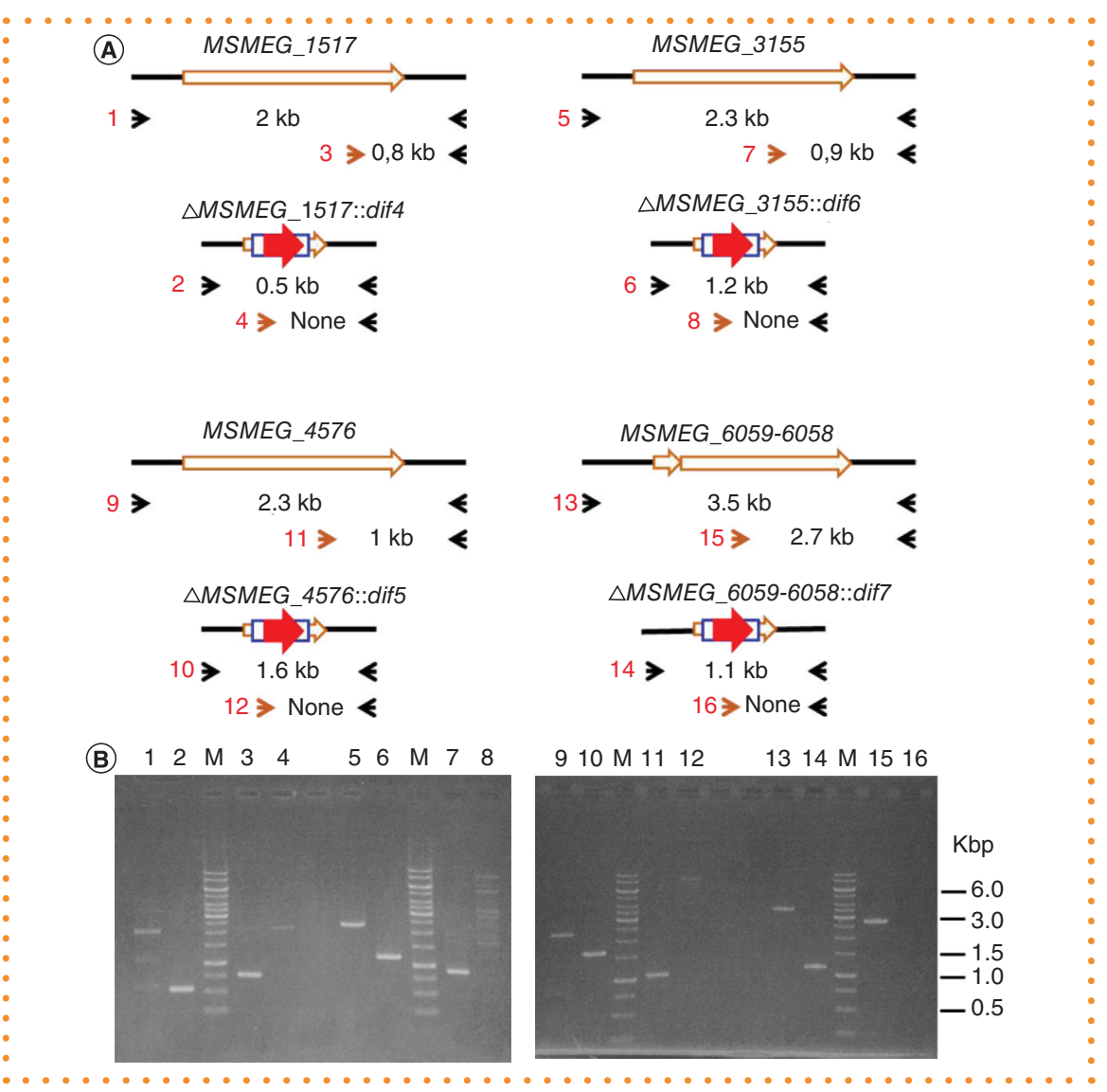




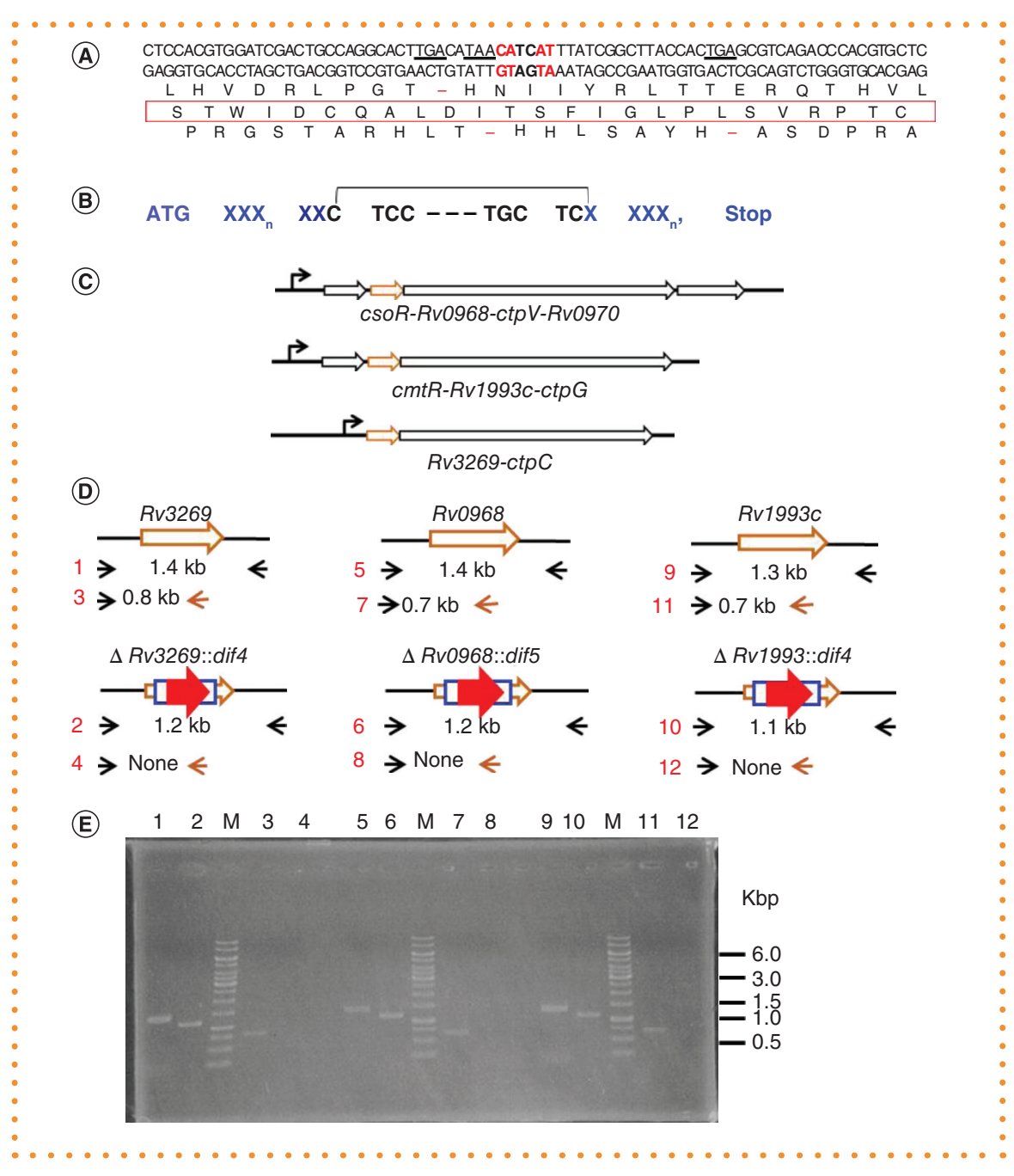

mentary Table 1), and a different dif variant for each gene to be deleted allowed construction of multiple deletions. For example, we constructed a quadruple mutant, mc ${ }^{2} 155 \Delta$ MSMEG_1517::dif4 $\triangle M S M E G$ 4576::dif5 $\triangle M$ MSMEG_3155::dif6 $\triangle M S M E G$ 6059-6058::dif7. DNA extracted from this mutant was amplified in PCR reactions with pairs of external primers or with an external primer and a second one internal to the deleted sequence (Figure $2 \mathrm{~A}$ \& Supplementary Table 1). Analysis of the PCR products confirmed the genetic structure of the mutant (Figure 2B).

\section{CONSTRUCTION OF MULTIPLE UNMARKED IN-FRAME DELETIONS IN M. TUBERCULOSIS}

Deletion of genes embedded within operons often results in polar effects on the downstream genes, and an interesting advantage of excisable cassettes is the possibility to construct in-frame deletions, which circumvent such effect [4,13]. After excision, the dif-Zeo ${ }^{\mathrm{R}}$-dif cassettes used here leave a 78-bp scar with no stop codon in the +1 translation frame (Figure 1E \& $3 \mathrm{~A})$. Therefore, provided that internal deletions of the target gene were designed in-frame with this +1 frame, the final construction fuses the translation start to the 26 aa translated from this frame and the last codons of the deleted gene (Figure 3B), which should not affect expression of the downstream genes. For example, the genes $\operatorname{ctp} C, c t p G$ and $c t p V$, encoding P-type ATPases involved in efflux of heavy metal ions in $M$. tuberculosis [14-16], are organized in operons containing an upstream gene, encoding a small protein of unknown function, Rv3269, Rv1993c or Rv0968, respectively (Figure 3C). A triple mutant of M. tuberculosis deleted of Rv0968, Rv1993c and Rv3269, namely H37Rv $\Delta$ Rv0968::dif5
Figure 3. Construction and verification of H37Rv $\Delta$ Rv0968::dif5 $\Delta$ Rv1993c::dif6 $\Delta$ Rv3269::dif4, a triple in-frame deletion mutant of $M$. tuberculosis. (A) Translation of the 78-bp dif4 scar left after cassette excision. Nucleotides in bold indicate the dif site central region. Nucleotides in red correspond to the dif 4 modifications. Stop codons in ORF 0 and +2 are underlined. (B) In-frame deletion resulting from fusion of the $5^{\prime}$ and $3^{\prime}$ ends of the gene of interest (blue nucleotides) to the +1 ORF of the dif-scar (black nucleotides and bracket). (C) Genetic organization of the operons encoding $c t p C$, ctpG and ctpV. (D) Schematics of the primers (arrowheads) and $\mathrm{PCR}$ products used to verify the three deletions. Brown arrowheads indicate primers internal to the sequences to be deleted. Red numbers in front of each pair of primers refer to the lanes in $3 \mathrm{E}$. (E) Agarose gel analysis of the amplified fragments. PCR were performed using DNA from wild-type H37Rv (odd numbers) or the triple mutant strain (even numbers).

M: 1 kb DNA ladder (Thermo Scientific), bands of higher intensity correspond to 1, 3 and $6 \mathrm{kbp}$.

$\Delta R v 1993 c::$ dif6 $\Delta$ Rv3269::dif4, was constructed in three rounds of recombineering with appropriate AES (Supplementary Table 2). The PCR verification of this triple mutant is shown in Figure 3E. Sequencing of the PCR fragments encompassing the variant dif sites confirmed the in-frame nature of the three deletions and complementation experiments (data not shown) confirmed that the CtpC efflux pump is still expressed in the $\Delta R v 3269::$ dif4 mutant.

In conclusion, we believe that the improvement of the Xer-cise technique described here can be useful to construct mutants in all mycobacterial species prone to recombineering. Our approach is applicable to any antibiotic resistance cassette by adapting the primers used to amplify the cassette (Table 2). It can also be extended to the many bacterial species in which XerCD, or the functionally equivalent XerS or XerH systems are present, 
and to archaeal species in which a Xer-like system operates $[17,18]$.

\section{SUPPLEMENTARY DATA}

To view the supplementary data that accompany this paper please visit the journal website at: www.future-science. com/doi/suppl/10.2144/btn-2019-0119

\section{AUTHOR CONTRIBUTIONS}

Y-M Boudehen, M Wallat and C Gutierrez performed experiments. O Neyrolles acquired funding. C Gutierrez and Y-M Boudehen wrote the manuscript; O Neyrolles and $\mathrm{P}$ Rousseau edited the manuscript. All the authors contributed to conception and design, analysis and interpretation of data.

\section{ACKNOWLEDGMENTS}

We thank $V$ Malaga and S Jamet for the gift of plasmids $\mathrm{pJV} 53 \mathrm{H}$ and $\mathrm{pGET}-\mathrm{Zeo}^{\mathrm{R}}{ }^{\text {, }}$, espectively, and F-X Barre and F Cornet for helpful discussions.

\section{FINANCIAL \& COMPETING INTERESTS DISCLOSURE}

This work was supported by Centre National de la Recherche Scientifique; Université Paul Sabatier; Agence Nationale de la Recherche (ANR14-CE14-0024), Fondation pour la Recherche Médicale (DEQ2016 0334902); Y-M Boudehen is supported by FRM. The authors have no other relevant affiliations or financial involvement with any organization or entity with a financial interest in or financial conflict with the subject matter or materials discussed in the manuscript apart from those disclosed.

No writing assistance was utilized in the production of this manuscript.

\section{OPEN ACCESS}

This work is licensed under the AttributionNonCommercial-NoDerivatives 4.0 Unported License. To view a copy of this license, visit http://creativecommons.org/licenses/ by-nc-nd/4.0/

\section{REFERENCES}

Papers of special note have been highlighted as: • of considerable interest

1. van Kessel JC, Hatfull GF. Recombineering in Mycobac terium tuberculosis. Nature Meth. 4, 147-152 (2007).

.. Describes recombineering, a phage recombinase-dependent technique allowing allelic exchange in Mycobacteria.

2. Mugweru J, Makafe G, Cao $\mathrm{Y}$ et al. A cassette containing thiostrepton, gentamicin resistance genes, and dif sequences is effective in construction of recombinant Mycobacteria. Front. Microbiol. 8, 468 (2017).

3. Bloor AE, Cranenburgh RM. An efficient method of selectable marker gene excision by Xer recombination for gene replacement in bacterial chromosomes. Appl. Environ. Microbiol. 72, 2520-2525 (2006).

- Describes the Xer-cise technique, using naturally present recombinases XerCD to excise antibiotic resistance cassettes flanked by tandem dif sites.

4. Cascioferro A, Boldrin F, Serafini A, Provvedi R, Manganelli R. Xer site-specific recombination, an efficient tool to introduce unmarked deletions into mycobacteria. Appl. Environ. Microbiol. 76, 5312-5316 (2010).

5. Mao XJ, Yan MY, Zhu H, Guo XP, Sun YC. Efficient and simple generation of multiple unmarked gene deletions in Mycobacterium smegmatis. Sci. Rep. 6, 22922 (2016).

6. Hendrickson H, Lawrence JG. Mutational bias suggests that replication termination occurs near the dif site, not at Ter sites. Mol. Microbiol. 64, 42-56 (2007).

7. Crozat E, Fournes F, Cornet F, Hallet B, Rousseau P. Resolution of multimeric forms of circular plasmids and chromosomes. Microbiol. Spectr. 2, PLAS-0025 (2014).

8. Grindley ND, Whiteson KL, Rice PA. Mechanisms of site-specific recombination. Ann. Rev. Biochem. 75, 567-605 (2006)

-. A thorough review on the mechanisms of site-specific recombinases.

9. Val ME, Kennedy SP, El Karoui M, Bonné L, Chevalier F, Barre FX. FtsK-dependent dimer resolution on multiple chromosomes in the pathogen Vibrio cholerae. PLOS Genet. 4, e1000201 (2008).

10. Cornet F, Mortier I, Patte J, Louarn JM. Plasmid pSC101 harbors a recombination site, $p s i$, which is able to resolve plasmid multimers and to substitute for the analogous chromosomal Escherichia coli site dif. J. Bacteriol. 176, 3188-3195 (1994).

11. Summers DK. Derivatives of ColE1 cer show altered topological specificity in site-specific recombination. EMBO J. 8, 309-315 (1989).

12. Jamet $\mathrm{S}$, Slama $\mathrm{N}$, Domingues $\mathrm{J}$ et al. The non-essential mycolic acid biosynthesis genes hadA and hadC contribute to the physiology and fitness of Mycobacterium smegmatis. PloS ONE 10, e0145883 (2015).

13. Marcus SA, Sidiropoulos SW, Steinberg H, Talaat AM. CsoR is essential for maintaining copper homeostasis in Mycobacterium tuberculosis. PloS One 11, e0151816 (2016).

14. Botella $\mathrm{H}$, Peyron $P$, Levillain $F$ et al. Mycobacterial p(1)-type ATPases mediate resistance to zinc poisoning in human macrophages. Cell Host Microbe. 10, 248-259 (2011).

15. Botella $H$, Stadthagen $G$, Lugo-Villarino $G$, de Chastellier $\mathrm{C}$, Neyrolles $\mathrm{O}$. Metallobiology of host-pathogen interac tions: an intoxicating new insight. Trends Microbiol. 20, 106-112 (2012)

16. Ward SK, Abomoelak B, Hoye EA, Steinberg $H$, Talaat AM. CtpV: a putative copper exporter required for full virulence of Mycobacterium tuberculosis. Mol. Microbiol. 77 , 1096-1110 (2010).

17. Castillo F, Benmohamed A, Szatmari G. Xer Site Specific Recombination: Double and Single Recombinase Systems. Front. Microbiol. 8, 453 (2017).

- An up-to-date review on the Xer and Xer-like systems in bacteria and archaea.

18. Debowski AW, Gauntlett JC, Li H et al. Xer-cise in Helicobacter pylori: one-step transformation for the construction of markerless gene deletions. Helicobacter. 17 435-443 (2012). 